\title{
The role of omega-3 in the prevention and treatment of sarcopenia
}

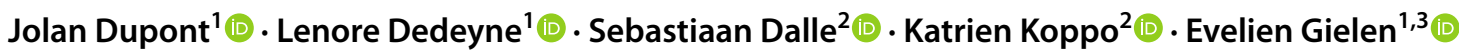

Received: 15 December 2018 / Accepted: 2 February 2019 / Published online: 19 February 2019

(c) The Author(s) 2019

\begin{abstract}
Sarcopenia is a geriatric syndrome with increasing importance due to the aging of the population. It is known to impose a major burden in terms of morbidity, mortality and socio-economic costs. Therefore, adequate preventive and treatment strategies are required. Progressive resistance training and protein supplementation are currently recommended for the prevention and treatment of sarcopenia. Omega-3 polyunsaturated fatty acids (PUFAs) might be an alternative therapeutic agent for sarcopenia due to their anti-inflammatory properties, which target the 'inflammaging', the age-related chronic low-grade inflammation which is assumed to contribute to the development of sarcopenia. In addition, omega-3 PUFAs may also have an anabolic effect on muscle through activation of the mTOR signaling and reduction of insulin resistance. This narrative review provides an overview of the current knowledge about omega-3 PUFAs and their role in the prevention and treatment of sarcopenia. We conclude that there is growing evidence for a beneficial effect of omega-3 PUFAs supplementation in sarcopenic older persons, which may add to the effect of exercise and/or protein supplementation. However, the exact dosage, frequency and use (alone or combined) in the treatment and prevention of sarcopenia still need further exploration.
\end{abstract}

Keywords Fatty acids $\cdot$ Omega-3 $\cdot$ Sarcopenia $\cdot$ Aged $\cdot$ Exercise training $\cdot$ Proteins

\section{Introduction}

Sarcopenia, the age-related loss of muscle mass and muscle strength, is a key feature of the aging process that predisposes elderly individuals to disability, immobility, falls, fractures and death [1]. Loss of muscle mass and strength strongly affects an older person's independence and quality of life [2]. The term 'sarcopenia' was first introduced by Rosenberg in 1997 to indicate the age-related loss of muscle mass [1]. Later on, several expert groups such as the International Working Group on Sarcopenia (IWGS) and the European Working Group on Sarcopenia in Older People (EWGSOP) expanded the definition with muscle function (muscle strength or physical performance) [2,3]. The

Evelien Gielen

evelien.gielen@uzleuven.be

1 Division of Gerontology and Geriatrics, Department of Chronic Diseases, Metabolism and Ageing (CHROMETA), KU Leuven, Leuven, Belgium

2 Exercise Physiology Research Group, Department of Movement Sciences, KU Leuven, Leuven, Belgium

3 Department of Geriatric Medicine, UZ Leuven, Herestraat 49, 3000 Leuven, Belgium
EWGSOP defined conceptual stages of sarcopenia, i.e., 'presarcopenia', 'sarcopenia' and 'severe sarcopenia'. To meet the criteria of sarcopenia, low muscle mass was required together with low muscle strength or low physical performance [2]. However, a consensus operational definition of sarcopenia is still lacking since the definitions of the expert groups propose different thresholds and diagnostic tests for the assessment of muscle mass, muscle strength and physical performance.

Recently, the EWGSOP revised its definition and diagnostic criteria for sarcopenia, placing muscle strength at the forefront instead of muscle mass (EWGSOP2) [4]. This new European consensus definition is in line with the growing evidence that muscle strength is better than muscle mass in predicting adverse outcomes [5]. EWGSOP2 also offers a clinically useful algorithm for case finding, diagnosis and confirmation of sarcopenia in older adults, defining new stages like 'probable sarcopenia', 'confirmed sarcopenia' and 'severe sarcopenia'. Finally, EWGSOP2 provides clear cutoff points for the measurement of muscle mass, muscle strength and physical performance. This, together with the introduction of the ICD-10 code for sarcopenia in 2016, which was necessary to identify sarcopenia as a disease, will hopefully lead to a greater interest of clinicians to diagnose 
sarcopenia and of the industry to develop new therapies for sarcopenia [6-9].

Sarcopenia has an important impact on daily life activities of elderly persons. For example, due to sarcopenia almost $20 \%$ of women and almost $10 \%$ of men $\geq 65$ years cannot lift a $4.5 \mathrm{~kg}$ weight or kneel down [10]. The condition is also found to be a significant predictor of hospitalization among older individuals [11]. Furthermore, the prevalence of sarcopenia is high, affecting up to $29 \%$ of community-dwelling adults aged $\geq 50$ years, up to $10 \%$ for those in acute hospital care and between 14 and 33\% of those living in longterm care institutions [12-14]. A recent longitudinal cohort study in 52 nursing homes in Belgium even suggests a higher prevalence with up to $45 \%$ of residents having sarcopenia [15]. Regardless of the applied definition, it goes without saying that sarcopenia represents an increasingly important public health concern. In 2000, the estimated healthcare cost attributable to sarcopenia in the United States was about $\$ 20$ billion, corresponding to $1.5 \%$ of the total healthcare expenditures [16]. These numbers are expected to increase with the aging of the population and the concomitant rise in the prevalence of sarcopenia. A recent systematic review indeed found higher healthcare costs for patients with sarcopenia, but concluded that more research should be conducted to assess the true impact of sarcopenia on healthcare consumption [17].

Physical exercise and nutritional interventions are currently recommended for the prevention and treatment of sarcopenia $[18,19]$. Progressive resistance training and protein and/or amino acids supplementation are generally accepted as the most effective approach to manage age-related muscle wasting $[12,20]$. In the elderly, however, the response to physical exercise and protein intake is blunted compared to younger persons, a phenomenon referred to as anabolic resistance [21]. To counteract this anabolic resistance, guidelines recommend a higher protein intake in the elderly ( $\geq 65$ years) as compared to the recommended daily allowance (RDA) for adults $<65$ years $(0.8 \mathrm{~g}$ protein $/ \mathrm{kg}$ of body weight (BW)/day). Healthy older persons are recommended to consume an average daily intake of $1.0-1.2 \mathrm{~g}$ protein $/ \mathrm{kg}$ BW or more $[18,22]$. Older adults who suffer from acute or chronic diseases require a daily protein intake of $1.2-1.5 \mathrm{~g} /$ $\mathrm{kg} \mathrm{BW}$, while severely ill older adults may need up to $2.0 \mathrm{~g}$ protein/kg BW per day. Another strategy to stimulate muscle growth in elderly, despite the occurrence of anabolic resistance, is progressive resistance training. Finally, there is growing interest in the combined effect of physical exercise and protein supplementation on gains in muscle mass and muscle function [23, 24]. Physical activity may restore the sensitivity of older muscles to protein intake and, in turn, the ingestion of sufficient proteins in close temporal proximity to exercise produces an additional anabolic stimulus that increases the post-exercise muscle protein synthesis [25].
Another approach for sarcopenia treatment might lie in targeting inflammation. Chronic low-grade inflammation associated with aging, also referred to as 'inflammaging', may be an important contributor to the development of sarcopenia [26, 27]. This increased inflammatory state may form a potential therapeutic target for sarcopenia. Antiinflammatory drugs might help to decrease inflammatory signaling and concomitantly improve physical performance in older adults [28]. Non-steroidal anti-inflammatory drugs (NSAIDs) such as piroxicam, celecoxib and ibuprofen have been tested in the past for this purpose but are currently not recommended for the treatment of sarcopenia due to the high risk on adverse events in the elderly [29-31]. Omega-3 polyunsaturated fatty acids (PUFAs) might be an alternative therapeutic agent for sarcopenia, with low risk on serious adverse events. Omega-3 PUFAs have anti-inflammatory properties and are generally known for their beneficial effects on cardiovascular risks, though a recent Cochrane review showed little or no effect of supplementation on cardiovascular health or mortality [32]. Furthermore, omega-3 PUFAs might also be beneficial for bone health, cognitive performance and eye health [33]. Finally, there is growing evidence that omega-3 PUFAs have an anabolic effect on skeletal muscle metabolism [34]. Therefore, omega-3 PUFAs supplementation may be a promising agent in the prevention and treatment of sarcopenia.

This narrative review provides an overview of the current knowledge about omega-3 PUFAs and their role in the prevention and treatment of sarcopenia. First, the age-related process of 'inflammaging' and the role of omega-3 PUFAs in this condition will be discussed. Next, we will clarify the mechanisms of action of omega-3 PUFAs, followed by an overview of the association between omega-3 PUFAs dietary intake, plasma levels and RBC content on the one hand and muscle mass, muscle strength and physical performance on the other hand. Finally, we will describe the results of interventional studies with omega-3 PUFAs supplementation.

\section{Inflammaging and its role in the development and progression of sarcopenia}

Aging is often accompanied by a slight increase in plasma levels of proinflammatory mediators such as interleukin 6 (IL-6), tumor necrosis factor alpha (TNF $\alpha$ ), c-reactive protein (CRP), interleukin 1 beta (IL-1 $\beta$ ), and reduced levels of anti-inflammatory cytokines such as interleukin 10 (IL10) [35]. This age-related chronic low-grade inflammation is referred to as immunosenescence or 'inflammaging' and involves a deteriorated immunity in elderly, making them more susceptible to infections and impeding the immune response upon infection [26]. 
The etiology of 'inflammaging' and its potential causal role in adverse health outcomes remains unclear, but most age-related diseases are associated with chronic low-grade inflammation [36]. It was suggested that increased plasma levels of proinflammatory cytokines might play a key role in the development and progression of sarcopenia, as these cytokines directly affect muscle catabolic and anabolic signaling pathways [37-40].

In their recent meta-analysis, Bano et al. concluded that sarcopenia is associated with elevated serum CRP levels, while there was no difference in IL-6 levels between sarcopenic and non-sarcopenic elderly [41]. In addition, other studies found an association between elevated CRP and sarcopenia or identified higher levels of CRP as a risk factor for the loss of muscle strength [38]. Accordingly, populationbased data suggest that levels of IL- 6 and TNF $\alpha$ are significantly elevated in Chinese sarcopenic elderly [40]. It can be concluded that data regarding inflammatory cytokines (especially IL-6) and the occurrence of sarcopenia are very limited and rather inconsistent.

IL-6 is presumed to play a dual role in the development and progression of sarcopenia. It was initially thought that this cytokine was only produced by immune cells, while later findings also identified muscle cells as IL- 6 producers. In fact, muscle is a major source of circulating IL-6, especially in response to exercise $[42,43]$. The role of IL-6 in the muscle remains controversial. On the one hand, when acutely increased, it might positively affect the muscle metabolism, e.g., by upregulation of the lipolysis and fat oxidation, while on the other hand it might increase the risk of sarcopenia, when chronically increased, as is the case in inflammaging.

\section{How to counteract this inflammaging?}

The exact pathway by which inflammation causes sarcopenia is still poorly understood, but lowering inflammation with anti-inflammatory agents might offer a window of opportunities to target sarcopenia.

NSAIDs might help to decrease inflammation and improve physical performance in older adults [28]. Piroxicam is shown to improve clinically relevant measures of muscle performance and mobility (e.g., fatigue resistance) in geriatric patients hospitalized with acute infection-induced inflammation [29]. Similarly, celecoxib has a beneficial effect on fatigue resistance in these acute ill older patients [30]. In healthy untrained older adults, aged 60-85 years, $1200 \mathrm{mg}$ ibuprofen a day significantly increases muscle hypertrophy in response to resistance exercise [31]. Because of the risk of adverse events (e.g., gastro-intestinal and renal toxicity and congestive heart failure) in older adults, the pros and cons of NSAIDs should be considered individually for each patient and their use is not yet recommended for this indication [30].

The effect of angiotensin converting enzyme inhibitors (ACE-inhibitors) on physical performance in the elderly has been examined, but data remain scarce and inconsistent [28]. Therefore, more research is needed to determine their role in the treatment of sarcopenia.

Omega-3 PUFAs supplementation might offer an interesting alternative opportunity, as it possesses anti-inflammatory properties and has a low risk of adverse events. Omega-3 PUFAs or n-3 PUFAs are characterized by the location of the first double bond at the third carbon. The main omega-3 PUFAs are $\alpha$-linolenic acid (ALA), eicosapentaenoic acid (EPA), docosapentaenoic acid (DPA) and docosahexaenoic acid (DHA). EPA, DPA and DHA are considered long-chain PUFAs, whereas ALA is considered short-chain PUFA [44]. The concentration of EPA and DHA in cell membranes is important for ensuring structural cell function [45, 46]. ALA can be metabolized into EPA and DPA in healthy young men and women to compensate for insufficient dietary intake. This is not the case for DHA, of which sufficient intake through the diet is crucial to maintain adequate membrane DHA concentrations [45, 47]. Data from elderly persons regarding ALA metabolizing into EPA and DPA were not found.

Fatty fish (e.g., salmon and mackerel) and seafood are the main food sources for EPA and DHA. Unfortunately, these food sources are often scarce in a Western diet [44]. ALA is more abundantly present in this diet, since it is found in plant foods like nuts, chia seeds and vegetable oils (e.g., soybean oil) [44]. The Academy of Nutrition and Dietetics recommends consuming two to three servings of fatty fish a week, aiming to provide about $500 \mathrm{mg}$ EPA and DHA each day. Observational research shows that most U.S. adults do not reach the recommended intake of omega-3 PUFAs, i.e., EPA and DHA. Elderly had higher intakes of EPA, but not DHA, compared to younger adults [48]. Therefore, EPA and DHA supplementation may be considered to reach the recommended daily intake.

Higher total omega-3 PUFAs plasma levels significantly reduce cardiovascular mortality, with a $35 \%$ lower risk on cardiovascular related death and an up to $69 \%$ lower risk for arrhythmic cardiovascular death [49]. Therefore, omega-3 PUFAs supplements were suggested for the prevention and treatment of cardiovascular diseases, if dietary intake is insufficient [50]. However, as mentioned before a recent Cochrane review showed inconsistent data about cardiovascular benefits of omega-3 PUFAs supplementation [32].

Omega-3 PUFAs supplementation might be beneficial for various aging processes, like cognition, bone health and eye health [33]. For example, omega-3 PUFAs supplementation reduces oxidative stress in healthy middle-aged to older U.S. adults, having a possible beneficial effect on 
cognition [51]. Furthermore, one study suggested that krill oil-derived omega-3 PUFAs can activate cognitive function in healthy older persons [52]. On the contrary, a larger study in 867 cognitively healthy elderly found no difference in cognitive function between elderly supplemented with either EPA + DHA or olive oil for 2 years. Thus, results are conflicting and further research is needed to confirm the effect of omega-3 PUFAs on cognition [53].

Several potential beneficial effects of omega-3 PUFAs on the muscle are suggested, like an increased nerve conduction and muscle activation, an improved mitochondrial function (energy production) or a larger myofiber size [54]. Furthermore, previous randomized controlled trials (RCTs) have shown that omega-3 PUFAs supplementation stimulates muscle protein synthesis in older adults $[55,56]$. When combined with resistance training, omega-3 PUFAs supplementation augmented the increase in muscle function and quality (strength per unit muscle area) achieved by resistance training [57]. Similarly, ALA supplementation combined with resistance training resulted in a greater increase of knee flexor thickness in elderly men [58].

\section{Role of omega-3 in the prevention and treatment of sarcopenia}

In the next paragraphs, we will describe the potential role of omega-3 in the prevention and treatment of sarcopenia. First, we will elaborate on the suggested working mechanism of omega-3 PUFAs. This will be followed by a discussion of the association between omega-3 PUFAs intake and muscle mass and function. Finally, current evidence about the effect of omega-3 PUFAs supplementation on sarcopenia components will be evaluated.

\section{Working mechanisms of omega-3 PUFAs}

The exact mechanisms of action of omega-3 PUFAs in sarcopenia are still under research [59]. We will highlight three hypothetic mechanisms and their supporting data: anti-inflammatory effects, mammalian target of rapamycin (mTOR) activation and reduction of insulin resistance (See Fig. 1).

Anti-inflammatory effects of omega-3 PUFAs are generally accepted. In a recent meta-analysis, Custodero et al. confirmed a reduction of CRP and IL-6 after supplementation with omega-3 PUFAs in middle-aged and older adults [60]. In addition, a recent RCT investigated the effect of EPA and DHA therapy on inflammation in older adults. The supplementation had a significant lowering effect on IL-6, IL-1 $\beta$ and TNF $\alpha$ levels after 4 weeks of use and even greater after 8 weeks [61]. As previously mentioned, chronic low-grade inflammation is suggested to play a role in the development of sarcopenia. Therefore, the suppression of this low-grade inflammation is commonly presumed to be one mechanism through which omega-3 PUFAs might counteract sarcopenia. On the contrary, Smith et al. found no effect of omega-3 PUFAs supplementation on CRP, TNF $\alpha$ and IL-6 [55]. Further research in (pre)sarcopenic older adults is needed to confirm whether omega-3 supplementation has an effect on sarcopenia by lowering pro-inflammatory cytokines.

Another suggested working mechanism is a transcriptionally regulated anabolic effect through activation of the mTOR pathway. The mTOR pathway plays a key role in many processes of cell growth, but is mostly important for skeletal muscle generation and muscle protein synthesis. Through its downstream regulators 4E-BP1 and S6K1, mTOR regulates muscle protein synthesis [62]. Further digression in the pathways on which mTOR has an effect does not belong to the purpose of this paper, but can be reviewed in the paper of Weigl [62]. Smith et al. found an increased activation of the mTOR-p70s6k signaling pathway in response to increased amino acid and insulin supply
Fig. 1 Working mechanisms of omega-3 PUFAs supplementation on sarcopenia parameters. mTORC1 mammalian target of rapamycin complex 1

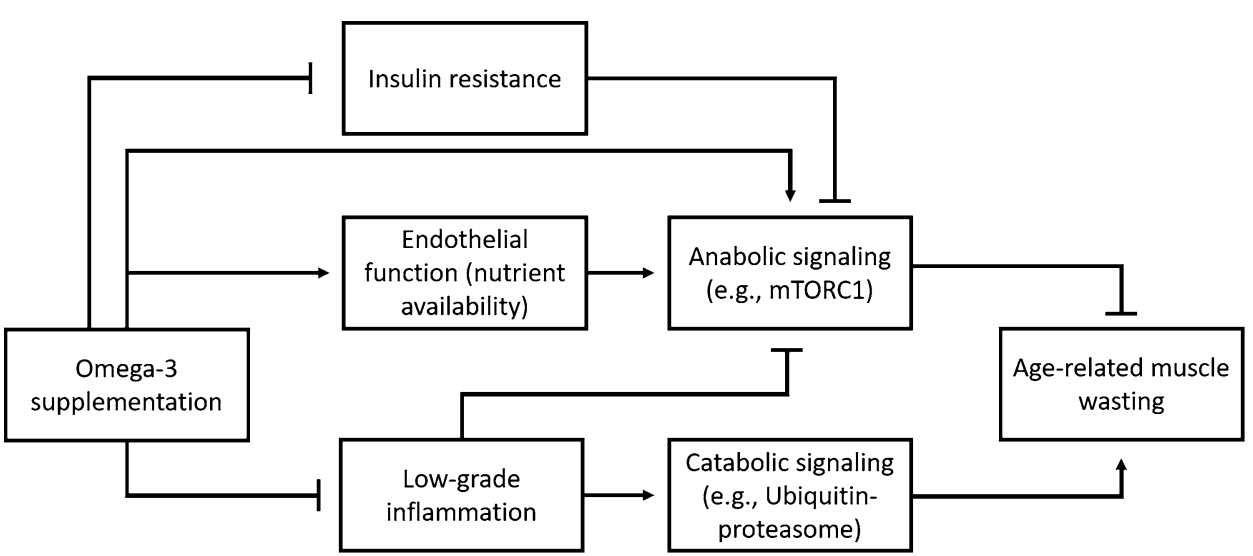


after 8 weeks of omega-3 PUFAs compared to a placebo group [55]. Yoshino et al. found that omega-3 PUFAs supplementation induces small but important changes in the gene expression, with an increased expression of pathways involving mitochondrial function regulation and a decreased expression of inhibitory pathways on mTOR, thus in favor of skeletal muscle anabolism [56, 63]. In conclusion, omega-3 PUFAs might help to overcome the age-related anabolic resistance by increasing the rate of muscle protein synthesis through stimulation of the mTOR signaling pathway.

Finally, some research suggests a role for decreased insulin resistance in response to omega-3 PUFA therapy [54, 64]. As insulin signaling plays a key role in mTOR activation, it is conceivable that omega-3 PUFA supplementation might alleviate the anabolic resistance and thus stimulate muscle protein synthesis in the elderly population [65]. However, further research is needed to elucidate the precise mechanism(s) through which omega-3 PUFAs affect insulin sensitivity.

\section{Association between omega-3 PUFAs intake and muscle mass and function}

Observational research suggests that a high intake of omega-3 PUFAs has a beneficial effect on physical performance in the elderly. Omega-3 PUFAs intake can be assessed through self-reported dietary intake or by measuring omega-3 PUFAs levels in plasma or red blood cell (RBC) membrane. In the next section, we will discuss the association between omega-3 PUFAs levels (assessed through self-reported dietary intake or by measuring omega-3 PUFAs plasma levels or RBC content) and sarcopenia components. The mentioned observational studies are summarized in Table 1.

Two cross-sectional studies have investigated the association between self-reported dietary intake of omega-3 fatty acids and physical performance. First, Robinson et al. examined the association between food intake and grip strength in 2893 men and women aged 59-73 years included in the Hertfordshire Cohort study. They found that each additional fatty fish consumption is associated with an increase in grip strength of $0.43 \mathrm{~kg}$ [95\% confidence interval (CI) 0.13-0.74] in men or $0.48 \mathrm{~kg}$ (95\% CI 0.24-0.72) in women [66]. Accordingly, Rousseau et al. reported a correlation between higher omega-3 PUFAs intake ( $>1.27 \mathrm{~g}$ daily) and increased leg strength or faster chair stand test. However, after partial correlations with protein as confounder, this association between omega-3 PUFAs intake and lower extremity function was no longer significant [67].

Omega-3 PUFAs plasma levels are often used as an indicator for dietary omega-3 intake, but results may vary due to a meal immediately prior to sample taking
[68]. Four studies were found using plasma levels of omega-3 PUFAs as a parameter of dietary intake. First, Frison et al. showed that among 982 older adults, those with a higher omega-3 PUFAs plasma level are less likely to have a lower gait speed $(<0.63 \mathrm{~m} / \mathrm{s})$ [69]. Secondly, Abbatecola et al. showed in a longitudinal study that lower baseline omega-3 PUFAs levels increase the risk of a decline in Short Physical Performance Battery (SPPB) score to $\leq 9$, compatible with low physical performance. Increased omega-3 PUFAs plasma levels seemed to protect against accelerated decline of physical performance, whereas a higher omega-6/omega-3 ratio was associated with increased risk of developing poor physical performance and slower gait speed [70]. This omega-6/omega-3 ratio is often used to assess cardiovascular risk, though the beneficial or harmful role of omega-6 PUFA remains uncertain [71, 72]. Third, a longitudinal study of Reinders et al. suggested that higher omega-3 PUFAs plasma levels, especially DHA, prevented a significant decrease in gait speed in women but not in men [73]. More research is needed to further elucidate whether omega-3 PUFAs might exert sex-specific effects. Another study of Reinders et al. described a cross-sectional association between higher concentrations of total PUFA and larger muscle size and greater knee extension strength. Remarkably, greater ALA levels were associated with decreased intermuscular adipose tissue, whereas EPA levels increased intermuscular adipose tissue [74]. Intermuscular adipose tissue is often used as a parameter for muscle quality and is independently associated with lower muscle strength $[75,76]$. The previously mentioned studies of Frison et al. and Abbatecola et al. did not investigate the amount of intermuscular adipose tissue, so further research is required to determine the association between omega-3 PUFAs plasma levels and intermuscular adipose tissue $[69,70]$.

As a parameter for omega-3 PUFAs intake researchers also often use the omega-3 index or red blood cell (RBC) membrane content of omega-3 PUFAs (mostly EPA and DHA) [77]. This can be used to measure the compliance with the intake of omega-3 PUFAs supplements [78, 79]. Only one study investigated the association between the omega-3 index and physical performance. Fougère et al. found that a low omega-3 index was associated with lower physical performance, measured by the SPPB in older adults with a mean age of 75 years. However, after confounder analysis for age, gender, grip strength, body mass index and geriatric depression scale, results were non-significant, indicating that the beneficial effect of omega-3 PUFAs on physical performance might be influenced by confounders [78].

Thus, observational data show that a higher omega-3 PUFAs intake or higher plasma levels/RBC content of omega-3 PUFAs are associated with higher muscle mass, 


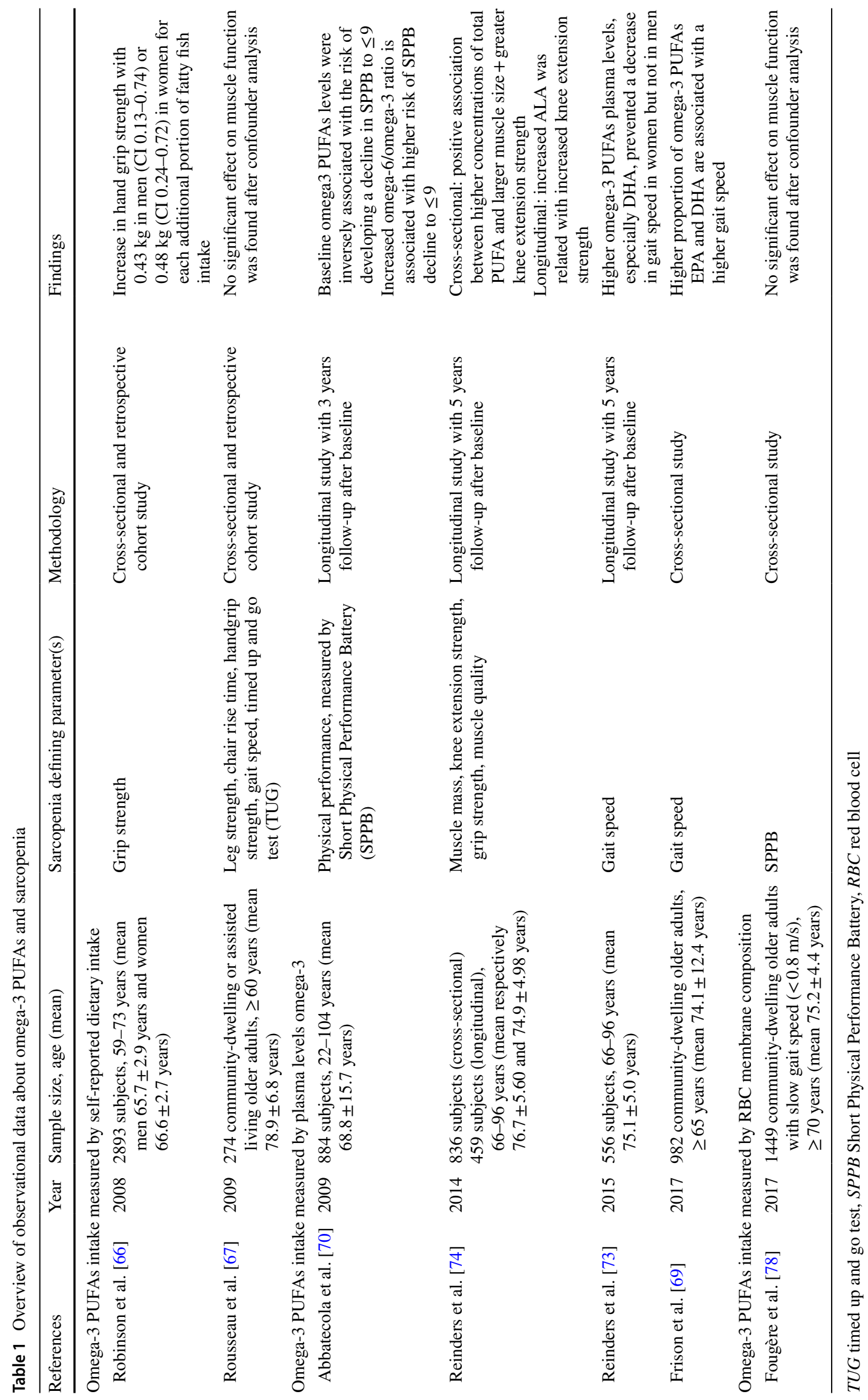


muscle strength, muscle quality and physical performance, all of which are important determinants for sarcopenia. In future research about sarcopenia, a systematic evaluation of omega-3 plasma or RBC levels might be useful as an indicator of omega-3 dietary intake.

\section{Effects of omega-3 PUFAs supplementation on muscle mass and muscle function}

To investigate the potential therapeutic role of omega-3 PUFAs supplementation in sarcopenic elderly, an increasing number of clinical trials have been and are being performed with omega-3 PUFAs supplements, either alone either in combination with an exercise intervention. This paper will discuss available RCTs and their results. We will start with RCTs investigating omega-3 PUFAs supplementation alone, followed by RCTs investigating a combination with exercise and finally discuss some data of the triple combination of omega-3 PUFAs supplementation, protein supplementation and exercise. An overview of these RCTs can be found in Table 2.

Four RCTs investigated the effect of omega-3 PUFAs supplementation alone in older adults. First, Hutchins-Wiese et al. found that 6 months of fish oil-derived supplementation (containing $1.2 \mathrm{~g}$ EPA and DHA a day) resulted in a significant increase in walking speed with $0.03-0.05 \mathrm{~m} / \mathrm{s}$ in postmenopausal women [80]. This small change is considered to be clinically relevant in the context of aging [81, 82]. Secondly, Smith et al. found that 8 weeks of omega-3 PUFAs supplementation (containing $1.86 \mathrm{~g}$ EPA and $1.5 \mathrm{~g}$ DHA a day) in healthy older adults ( $\geq 65$ years) increased the muscle protein synthesis in response to a hyperaminoacidemic-hyperinsulinemic clamp to a larger extend than compared to a corn oil supplemented group [55]. This observation suggests that omega-3 PUFAs supplementation improved the sensitivity to an anabolic stimulus (i.e., amino acids and insulin) and decreased the age-related anabolic resistance. The same research group demonstrated that 6 months omega-3 PUFAs supplementation (again containing $1.86 \mathrm{~g}$ EPA and $1.5 \mathrm{~g}$ DHA a day) significantly increased thigh muscle volume and muscle strength compared to a placebo in healthy older adults (60-85 years) [56]. Finally, Krzymińska-Siemaszko et al. could not demonstrate an effect of 12 weeks of omega-3 PUFAs supplementation (containing $660 \mathrm{mg}$ EPA and $440 \mathrm{mg}$ DHA a day) on muscle mass, muscle strength or gait speed in Polish older adults suffering from a decreased muscle mass [83]. The authors attribute this to the low sample size of their study and the lower dosage of EPA and DHA compared to the dosages used in the aforementioned studies of Hutchins-Wiese et al. and Smith et al. [55, 56, 80]. Additionally, duration of supplementation and characteristics of the study population might also contribute to the conflicting results.
Overall, the effect of omega-3 PUFAs supplements alone seems promising for sarcopenia by enhancing muscle protein synthesis, improving gait speed and increasing muscle strength and physical performance.

Furthermore, increasing evidence suggests that omega-3 PUFAs supplementation amplifies the effect of physical exercise on parameters of muscle mass, strength and performance. Three studies investigated the combined effect of omega-3 supplementation and an exercise intervention. In 2009, Cornish and Chilibeck combined 12 weeks of ALA supplementation with a resistance training program (three times a week) in older adults (mean age $65.4 \pm 0.8$ years) [58]. Muscle thickness of the elbow flexor, elbow extensor and knee extensor as well as chess and leg press strength significantly increased following resistance training, but no differences were observed between the ALA and the placebo group. The only benefit of additional ALA supplementation was a significantly larger increase in knee flexor muscle thickness. Secondly, Rodacki et al. randomly assigned healthy older women (aged $64 \pm 1.4$ years) to three groups: 90 days of strength training only, strength training combined with 90 days of fish oil supplementation or strength training combined with 150 days of supplementation (starting 60 days before intervention) [84]. The different duration of supplementation was to investigate whether the duration of supplementation modifies the effect. Strength training increased muscle strength in all groups, however, when combined with omega-3 PUFA supplements the effect on muscle strength and functional capacity was enhanced. There was no significant difference in effect between 90 days or 150 days of omega-3 PUFAs supplementation. Third, Da Boit et al. examined the effect of a resistance training program with omega-3 PUFAs supplements [57]. Older men and women (aged $\geq 65$ years) received supplements or placebo for 18 weeks, whilst performing a lower-limb resistance training twice weekly. Omega-3 PUFAs supplementation significantly augmented the increase in muscle function and quality (strength per unit muscle area) achieved by resistance training in older women, but not in men.

Combining omega-3 supplements with an exercise intervention might be a promising therapeutic agent for sarcopenia, with omega-3 supplements enhancing the effects of the exercise intervention on muscle mass and muscle function.

Up to now, only two studies have examined the combined effect of omega-3 PUFAs, exercise and protein supplements, though one of them in patients with chronic obstructive pulmonary disease (COPD) [85]. However, the supplement did not only consist of omega-3 PUFAs, so the specific omega 3-PUFA effect remains unclear. Recently Zhu et al. published a study in Chinese sarcopenic elderly, combining a training program (twice a week in-group, once a week at home) and a nutrition supplement twice daily for 12 weeks [86]. The supplement contained $8.61 \mathrm{~g}$ protein, 


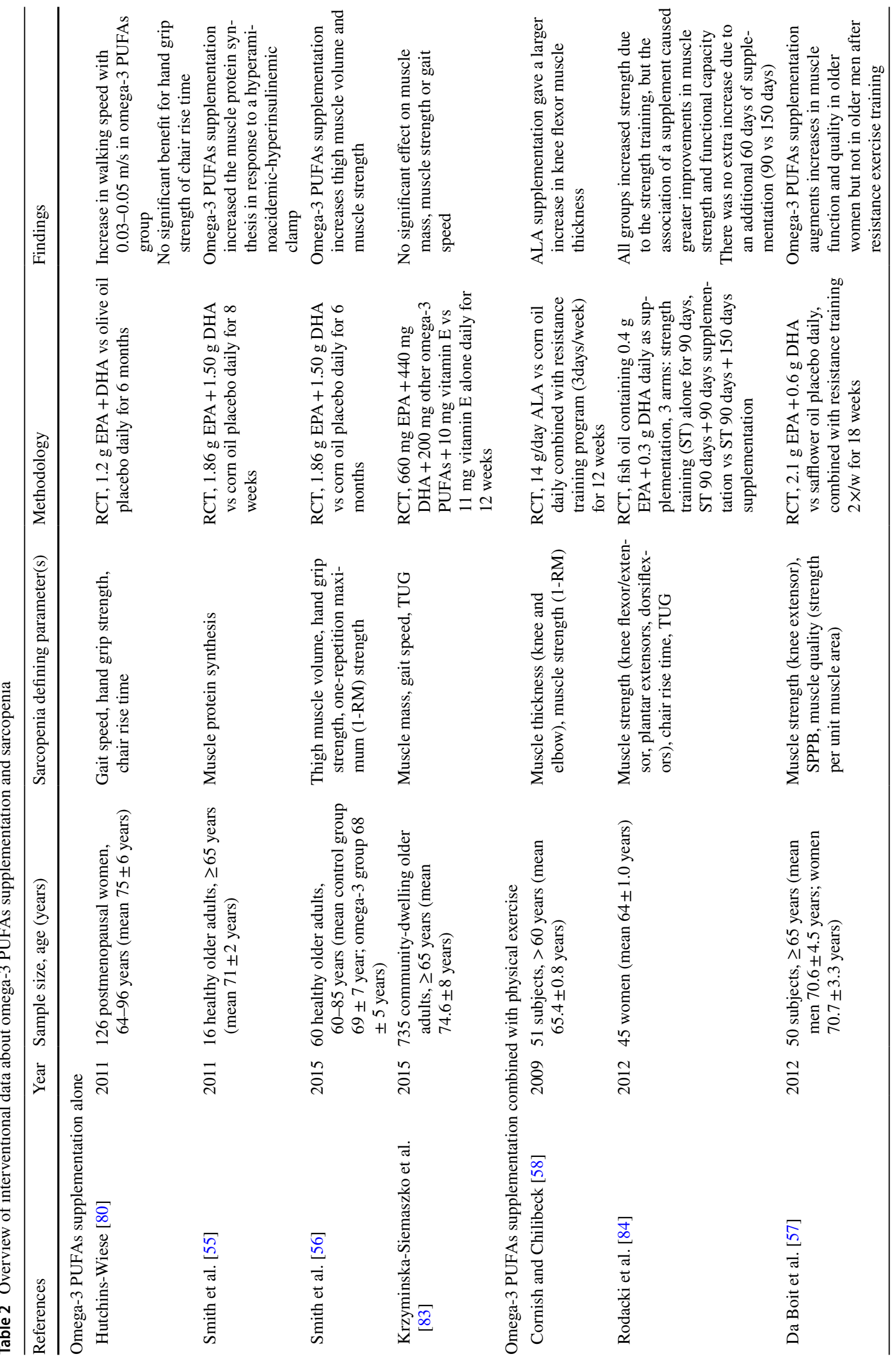




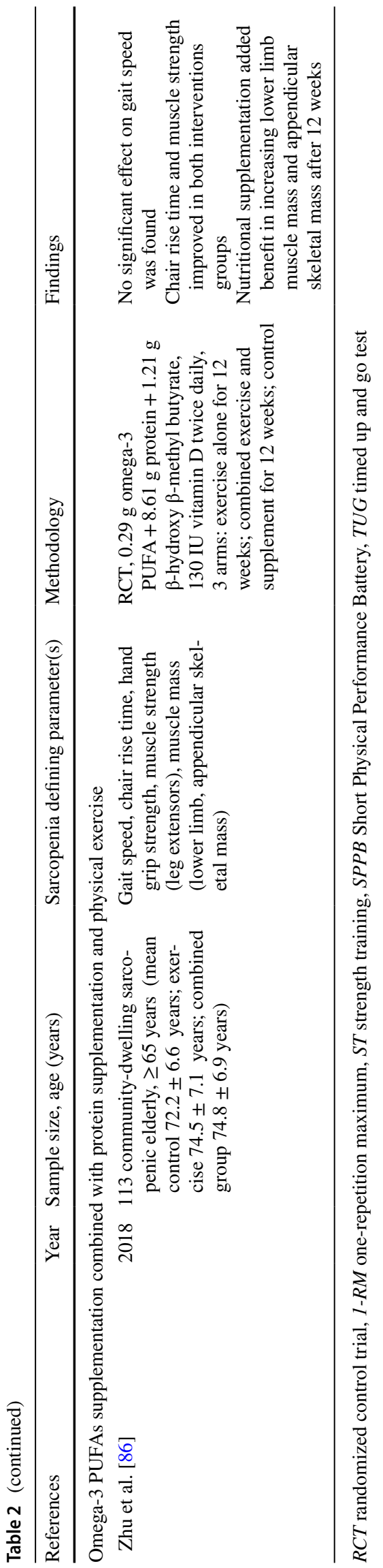

$1.21 \mathrm{~g} \beta$-hydroxy $\beta$-methyl butyrate, $130 \mathrm{IU}$ vitamin $\mathrm{D}$ and $0.29 \mathrm{~g}$ omega-3 PUFAs. When the nutritional supplement was combined with the exercise intervention, the authors found an additional effect on appendicular skeletal mass and lower limb muscle mass, compared to the exercise intervention alone. However, the authors acknowledge the very low dosage of omega-3 PUFAs. Therefore, it is unclear whether omega-3 PUFAs did contribute to the positive effects. In sarcopenic older persons, there are currently no other data available about the effect of the combination of protein supplementation, exercise and omega-3 PUFAs supplementation.

\section{Conclusion}

Sarcopenia is a geriatric syndrome with increasing importance due to the aging of the population. It is known to impose a major burden in terms of morbidity, mortality and socio-economic costs. Therefore, adequate preventive and treatment strategies are required. Progressive resistance training and protein supplementation are currently recommended for the prevention and treatment of sarcopenia.

Inflammaging may play an important role in the development of sarcopenia. This might offer a new target for the prevention and treatment of sarcopenia. Omega-3 PUFAs, which lower inflammation but which also may enhance mTOR signaling and reduce insulin resistance, are positively associated with muscle mass, muscle strength, muscle quality and physical performance. Therefore, omega-3 PUFAs supplements may be a potential therapy or preventive measure for sarcopenia, either alone or combined with the classic therapeutic strategies. The effect of omega-3 PUFAs supplements alone seems promising for sarcopenia by improving muscle mass, muscle strength and physical performance. In combination with an exercise intervention, omega-3 PUFAs supplementation might augment the increase in muscle mass and function obtained by the exercise intervention. The triple combination of exercise, protein and omega-3 PUFAs supplementation needs more research, especially in persons with sarcopenia, but omega-3 PUFAs might be a promising enhancer of the effects of physical exercise and protein supplementation. More research is needed to confirm these findings.

We conclude that there is growing evidence for a beneficial effect of omega-3 PUFAs supplementation in sarcopenic older persons. However, the exact dosage, frequency and use (alone or combined) in the treatment and prevention of sarcopenia still need further exploration. 


\section{Compliance with ethical standards}

Conflict of interest JD, LD, SD and KK have no conflicts of interest to declare. EG reports consultancy fees for UCB, Alexion and Takeda and speakers fees for Amgen and Takeda.

Statement of human and animal rights This article does not contain any studies with human participants or animals performed by any of the authors.

Informed consent For this type of study, formal consent is not required.

OpenAccess This article is distributed under the terms of the Creative Commons Attribution 4.0 International License (http://creativeco mmons.org/licenses/by/4.0/), which permits unrestricted use, distribution, and reproduction in any medium, provided you give appropriate credit to the original author(s) and the source, provide a link to the Creative Commons license, and indicate if changes were made.

\section{References}

1. Rosenberg IH (1997) Sarcopenia: origins and clinical relevance. J Nutr 127:990s-991s

2. Cruz-Jentoft AJ, Baeyens JP, Bauer JM et al (2010) Sarcopenia: European consensus on definition and diagnosis: report of the European Working Group on Sarcopenia in Older People. Age Ageing 39:412-423

3. Fielding RA, Vellas B, Evans WJ et al (2011) Sarcopenia: an undiagnosed condition in older adults. Current consensus definition: prevalence, etiology, and consequences. International working group on sarcopenia. J Am Med Dir Assoc 12:249-256

4. Cruz-Jentoft AJ, Bahat G, Bauer J et al (2018) Sarcopenia: revised European consensus on definition and diagnosis. Age Ageing 48:16-31

5. Schaap LA, van Schoor NM, Lips P et al (2018) Associations of sarcopenia definitions, and their components, with the incidence of recurrent falling and fractures: the Longitudinal Aging Study Amsterdam. J Gerontol A Biol Sci Med Sci 73:1199-1204

6. Anker SD, Morley JE, von Haehling S (2016) Welcome to the ICD-10 code for sarcopenia. J Cachexia Sarcopenia Muscle 7:512-514

7. Vellas B, Fielding RA, Bens C et al (2018) Implications of ICD10 for Sarcopenia Clinical Practice and Clinical Trials: report by the International Conference on Frailty and Sarcopenia Research Task Force. J Frailty Aging 7:2-9

8. Bahat G, Tufan A, Tufan F et al (2016) Suggestions on sarcopeniarelated trials. Aging Clin Exp Res 28:1029-1030

9. Blasimme A (2017) Physical frailty, sarcopenia, and the enablement of autonomy: philosophical issues in geriatric medicine. Aging Clin Exp Res 29:59-63

10. McLeod M, Breen L, Hamilton DL et al (2016) Live strong and prosper: the importance of skeletal muscle strength for healthy ageing. Biogerontology 17:497-510

11. Zhang X, Zhang W, Wang C et al (2018) Sarcopenia as a predictor of hospitalization among older people: a systematic review and meta-analysis. BMC Geriatr 18:188

12. Cruz-Jentoft AJ, Landi F, Schneider SM et al (2014) Prevalence of and interventions for sarcopenia in ageing adults: a systematic review. Report of the International Sarcopenia Initiative (EWGSOP and IWGS). Age Ageing 43:748-759

13. Landi F, Liperoti R, Fusco D et al (2012) Prevalence and risk factors of sarcopenia among nursing home older residents. J Gerontol A Biol Sci Med Sci 67:48-55
14. Gariballa S, Alessa A (2013) Sarcopenia: prevalence and prognostic significance in hospitalized patients. Clin Nutr 32:772-776

15. Perkisas S, De Cock AM, Vandewoude M et al (2018) Prevalence of sarcopenia and 9-year mortality in nursing home residents. Aging Clin Exp Res. https://doi.org/10.1007/s40520-018-1038-2

16. Janssen I, Shepard DS, Katzmarzyk PT et al (2004) The healthcare costs of sarcopenia in the United States. J Am Geriatr Soc 52:80-85

17. Bruyere O, Beaudart C, Ethgen O et al (2019) The health economics burden of sarcopenia: a systematic review. Maturitas 119:61-69

18. Bauer J, Biolo G, Cederholm T et al (2013) Evidence-based recommendations for optimal dietary protein intake in older people: a position paper from the PROT-AGE Study Group. J Am Med Dir Assoc 14:542-559

19. Beaudart C, Dawson A, Shaw SC et al (2017) Nutrition and physical activity in the prevention and treatment of sarcopenia: systematic review. Osteoporos Int 28:1817-1833

20. Beaudart C, Rabenda V, Simmons M et al (2018) Effects of protein, essential amino acids, B-hydroxy B-methylbutyrate, creatine, dehydroepiandrosterone and fatty acid supplementation on muscle mass, muscle strength and physical performance in older people aged 60 years and over. A systematic review on the literature. J Nutr Health Aging 22:117-130

21. Kumar V, Selby A, Rankin D et al (2009) Age-related differences in the dose-response relationship of muscle protein synthesis to resistance exercise in young and old men. J Physiol 587:211-217

22. Traylor DA, Gorissen SHM, Phillips SM (2018) Perspective: protein requirements and optimal intakes in aging: are we ready to recommend more than the recommended daily allowance? Adv Nutr 9:171-182

23. Marzetti E, Calvani R, Tosato M et al (2017) Sarcopenia: an overview. Aging Clin Exp Res 29:11-17

24. Dedeyne L, Deschodt M, Verschueren S et al (2017) Effects of multi-domain interventions in (pre)frail elderly on frailty, functional, and cognitive status: a systematic review. Clin Interv Aging 12:873-896

25. Pennings B, Koopman R, Beelen M et al (2011) Exercising before protein intake allows for greater use of dietary protein-derived amino acids for de novo muscle protein synthesis in both young and elderly men. Am J Clin Nutr 93:322-331

26. Franceschi C, Bonafe $M$, Valensin $S$ et al (2000) Inflamm-aging. An evolutionary perspective on immunosenescence. Ann N Y Acad Sci 908:244-254

27. Dalle S, Rossmeislova L, Koppo K (2017) The role of inflammation in age-related sarcopenia. Front Physiol 8:1045

28. Alturki M, Beyer I, Mets T et al (2018) Impact of drugs with anti-inflammatory effects on skeletal muscle and inflammation: a systematic literature review. Exp Gerontol 114:33-49

29. Beyer I, Bautmans I, Njemini R et al (2011) Effects on muscle performance of NSAID treatment with piroxicam versus placebo in geriatric patients with acute infection-induced inflammation. A double blind randomized controlled trial. BMC Musculoskelet Disord 12:292

30. Mets T, Bautmans I, Njemini R et al (2004) The influence of celecoxib on muscle fatigue resistance and mobility in elderly patients with inflammation. Am J Geriatr Pharmacother 2:230-238

31. Trappe TA, Carroll CC, Dickinson JM et al (2011) Influence of acetaminophen and ibuprofen on skeletal muscle adaptations to resistance exercise in older adults. Am J Physiol Regul Integr Comp Physiol 300:R655-R662

32. Abdelhamid AS, Brown TJ, Brainard JS et al (2018) Omega-3 fatty acids for the primary and secondary prevention of cardiovascular disease. Cochrane Database Syst Rev 11:Cd003177 
33. Ruxton CH, Derbyshire E, Toribio-Mateas M (2016) Role of fatty acids and micronutrients in healthy ageing: a systematic review of randomised controlled trials set in the context of European dietary surveys of older adults. J Hum Nutr Diet 29:308-324

34. Robinson SM, Reginster JY, Rizzoli R et al (2018) Does nutrition play a role in the prevention and management of sarcopenia? Clin Nutr 37:1121-1132

35. Baylis D, Bartlett DB, Patel HP et al (2013) Understanding how we age: insights into inflammaging. Longev Healthspan 2:8

36. Franceschi C, Campisi J (2014) Chronic inflammation (inflammaging) and its potential contribution to age-associated diseases. J Gerontol A Biol Sci Med Sci 69:S4-S9

37. Payette H, Roubenoff R, Jacques PF et al (2003) Insulin-like growth factor- 1 and interleukin 6 predict sarcopenia in very old community-living men and women: the Framingham Heart Study. J Am Geriatr Soc 51:1237-1243

38. Schaap LA, Pluijm SM, Deeg DJ et al (2006) Inflammatory markers and loss of muscle mass (sarcopenia) and strength. Am J Med 119:526.e9-526.e17

39. Schaap LA, Pluijm SM, Deeg DJ et al (2009) Higher inflammatory marker levels in older persons: associations with 5-year change in muscle mass and muscle strength. J Gerontol A Biol Sci Med Sci 64:1183-1189

40. Bian AL, Hu HY, Rong YD et al (2017) A study on relationship between elderly sarcopenia and inflammatory factors IL-6 and TNF-alpha. Eur J Med Res 22:25

41. Bano G, Trevisan C, Carraro S et al (2017) Inflammation and sarcopenia: a systematic review and meta-analysis. Maturitas 96:10-15

42. Karstoft K, Pedersen BK (2016) Skeletal muscle as a gene regulatory endocrine organ. Curr Opin Clin Nutr Metab Care 19:270-275

43. Benatti FB, Pedersen BK (2015) Exercise as an anti-inflammatory therapy for rheumatic diseases-myokine regulation. Nat Rev Rheumatol 11:86-97

44. Vannice G, Rasmussen H (2014) Position of the academy of nutrition and dietetics: dietary fatty acids for healthy adults. J Acad Nutr Diet 114:136-153

45. Burdge GC, Jones AE, Wootton SA (2002) Eicosapentaenoic and docosapentaenoic acids are the principal products of alphalinolenic acid metabolism in young men. Br J Nutr 88:355-363

46. Calder PC (1999) Dietary fatty acids and the immune system. Lipids 34:S137-S140

47. Burdge GC, Wootton SA (2002) Conversion of alpha-linolenic acid to eicosapentaenoic, docosapentaenoic and docosahexaenoic acids in young women. Br J Nutr 88:411-420

48. Papanikolaou Y, Brooks J, Reider C et al (2014) U.S. adults are not meeting recommended levels for fish and omega-3 fatty acid intake: results of an analysis using observational data from NHANES 2003-2008. Nutr J 13:31

49. Mozaffarian D, Lemaitre RN, King IB et al (2013) Plasma phospholipid long-chain omega-3 fatty acids and total and cause-specific mortality in older adults: a cohort study. Ann Intern Med 158:515-525

50. Sokola-Wysoczanska E, Wysoczanski T, Wagner J et al (2018) Polyunsaturated fatty acids and their potential therapeutic role in cardiovascular system disorders - a review. Nutrients 10:1561

51. Kiecolt-Glaser JK, Epel ES, Belury MA et al (2013) Omega-3 fatty acids, oxidative stress, and leukocyte telomere length: a randomized controlled trial. Brain Behav Immun 28:16-24

52. Konagai C, Yanagimoto K, Hayamizu K et al (2013) Effects of krill oil containing $n-3$ polyunsaturated fatty acids in phospholipid form on human brain function: a randomized controlled trial in healthy elderly volunteers. Clin Interv Aging 8:1247-1257

53. Dangour AD, Allen E, Elbourne D et al (2010) Effect of 2-y n-3 long-chain polyunsaturated fatty acid supplementation on cognitive function in older people: a randomized, double-blind, controlled trial. Am J Clin Nutr 91:1725-1732

54. Gray SR, Mittendorfer B (2018) Fish oil-derived n-3 polyunsaturated fatty acids for the prevention and treatment of sarcopenia. Curr Opin Clin Nutr Metab Care 21:104-109

55. Smith GI, Atherton P, Reeds DN et al (2011) Dietary omega-3 fatty acid supplementation increases the rate of muscle protein synthesis in older adults: a randomized controlled trial. Am J Clin Nutr 93:402-412

56. Smith GI, Julliand S, Reeds DN et al (2015) Fish oil-derived n-3 PUFA therapy increases muscle mass and function in healthy older adults. Am J Clin Nutr 102:115-122

57. Da Boit M, Sibson R, Sivasubramaniam S et al (2017) Sex differences in the effect of fish-oil supplementation on the adaptive response to resistance exercise training in older people: a randomized controlled trial. Am J Clin Nutr 105:151-158

58. Cornish SM, Chilibeck PD (2009) Alpha-linolenic acid supplementation and resistance training in older adults. Appl Physiol Nutr Metab 34:49-59

59. Di Girolamo FG, Situlin R, Mazzucco S et al (2014) Omega-3 fatty acids and protein metabolism: enhancement of anabolic interventions for sarcopenia. Curr Opin Clin Nutr Metab Care $17: 145-150$

60. Custodero C, Mankowski RT, Lee SA et al (2018) Evidencebased nutritional and pharmacological interventions targeting chronic low-grade inflammation in middle-age and older adults: a systematic review and meta-analysis. Ageing Res Rev 46:42-59

61. Tan A, Sullenbarger B, Prakash R et al (2018) Supplementation with eicosapentaenoic acid and docosahexaenoic acid reduces high levels of circulating proinflammatory cytokines in aging adults: a randomized, controlled study. Prostaglandins Leukot Essent Fatty Acids 132:23-29

62. Weigl LG (2012) Lost in translation: regulation of skeletal muscle protein synthesis. Curr Opin Pharmacol 12:377-382

63. Yoshino J, Smith GI, Kelly SC et al (2016) Effect of dietary n-3 PUFA supplementation on the muscle transcriptome in older adults. Physiol Rep 4:e12785

64. Albert BB, Derraik JG, Brennan CM et al (2014) Higher omega-3 index is associated with increased insulin sensitivity and more favourable metabolic profile in middle-aged overweight men. Sci Rep 4:6697

65. Bodine SC, Baehr LM (2014) Skeletal muscle atrophy and the E3 ubiquitin ligases MuRF1 and MAFbx/atrogin-1. Am J Physiol Endocrinol Metab 307:E469-E484

66. Robinson SM, Jameson KA, Batelaan SF et al (2008) Diet and its relationship with grip strength in community-dwelling older men and women: the Hertfordshire cohort study. J Am Geriatr Soc 56:84-90

67. Rousseau JH, Kleppinger A, Kenny AM (2009) Self-reported dietary intake of omega- 3 fatty acids and association with bone and lower extremity function. J Am Geriatr Soc 57:1781-1788

68. Harris WS, Thomas RM (2010) Biological variability of blood omega-3 biomarkers. Clin Biochem 43:338-340

69. Frison E, Boirie Y, Peuchant E et al (2017) Plasma fatty acid biomarkers are associated with gait speed in community-dwelling older adults: the Three-City-Bordeaux study. Clin Nutr 36:416-422

70. Abbatecola AM, Cherubini A, Guralnik JM et al (2009) Plasma polyunsaturated fatty acids and age-related physical performance decline. Rejuvenation Res 12:25-32

71. Harris WS (2006) The omega-6/omega-3 ratio and cardiovascular disease risk: uses and abuses. Curr Atheroscler Rep 8:453-459

72. Ramsden CE, Zamora D, Leelarthaepin B et al (2013) Use of dietary linoleic acid for secondary prevention of coronary heart 
disease and death: evaluation of recovered data from the Sydney Diet Heart Study and updated meta-analysis. BMJ 346:e8707

73. Reinders I, Murphy RA, Song X et al (2015) Polyunsaturated fatty acids in relation to incident mobility disability and decline in gait speed; the age, gene/environment susceptibility-Reykjavik study. Eur J Clin Nutr 69:489-493

74. Reinders I, Song X, Visser M et al (2015) Plasma phospholipid PUFAs are associated with greater muscle and knee extension strength but not with changes in muscle parameters in older adults. J Nutr 145:105-112

75. Goodpaster BH, Carlson CL, Visser M et al (2001) Attenuation of skeletal muscle and strength in the elderly: the Health ABC Study. J Appl Physiol (1985) 90:2157-2165

76. Reinders I, Murphy RA, Brouwer IA et al (2016) Muscle quality and myosteatosis: novel associations with mortality risk: the age, gene/environment susceptibility (AGES)-Reykjavik study. Am J Epidemiol 183:53-60

77. Witte TR, Salazar AJ, Ballester OF et al (2010) RBC and WBC fatty acid composition following consumption of an omega 3 supplement: lessons for future clinical trials. Lipids Health Dis 9:31

78. Fougere B, de Souto Barreto P, Goisser S et al (2018) Red blood cell membrane omega-3 fatty acid levels and physical performance: Cross-sectional data from the MAPT study. Clin Nutr 37:1141-1144

79. Gadaria-Rathod N, Dentone PG, Peskin E et al (2013) Red blood cell fatty acid analysis for determining compliance with omega3 supplements in dry eye disease trials. J Ocul Pharmacol Ther 29:837-841

80. Hutchins-Wiese HL, Kleppinger A, Annis K et al (2013) The impact of supplemental n-3 long chain polyunsaturated fatty acids and dietary antioxidants on physical performance in postmenopausal women. J Nutr Health Aging 17:76-80

81. Kwon S, Perera S, Pahor M et al (2009) What is a meaningful change in physical performance? Findings from a clinical trial in older adults (the LIFE-P study). J Nutr Health Aging 13:538-544

82. Perera S, Mody SH, Woodman RC et al (2006) Meaningful change and responsiveness in common physical performance measures in older adults. J Am Geriatr Soc 54:743-749

83. Krzyminska-Siemaszko R, Czepulis N, Lewandowicz M et al (2015) The effect of a 12-week omega-3 supplementation on body composition, muscle strength and physical performance in elderly individuals with decreased muscle mass. Int J Environ Res Public Health 12:10558-10574

84. Rodacki CL, Rodacki AL, Pereira G et al (2012) Fish-oil supplementation enhances the effects of strength training in elderly women. Am J Clin Nutr 95:428-436

85. Sugawara K, Takahashi H, Kashiwagura T et al (2012) Effect of anti-inflammatory supplementation with whey peptide and exercise therapy in patients with COPD. Respir Med 106:1526-1534

86. Zhu LY, Chan R, Kwok T et al (2018) Effects of exercise and nutrition supplementation in community-dwelling older Chinese people with sarcopenia: a randomized controlled trial. Age Ageing. https://doi.org/10.1093/ageing/afy 179

Publisher's Note Springer Nature remains neutral with regard to jurisdictional claims in published maps and institutional affiliations. 